DEMOGRAPHIC RESEARCH

VOLUME 41, ARTICLE 4, PAGES 83-102 PUBLISHED 9 JULY 2019

http://www.demographic-research.org/Volumes/Vol41/4/

DOI: 10.4054/DemRes.2019.41.4

Formal Relationship 29

\title{
The threshold age of the lifetable entropy
}

José Manuel Aburto

Jesús-Adrián Alvarez

Francisco Villavicencio

James W. Vaupel

(c) 2019 Aburto, Alvarez, Villavicencio \& Vaupel.

This open-access work is published under the terms of the Creative Commons Attribution 3.0 Germany (CC BY 3.0 DE), which permits use, reproduction, and distribution in any medium, provided the original author(s) and source are given credit.

See https://creativecommons.org/licenses/by/3.0/de/legalcode 


\section{Contents}

$1 \quad$ Relationship $\quad 84$

2 Proof $\quad 85$

$2.1 \quad$ Relative changes over time in $\bar{H} \quad 85$

$\begin{array}{lll}2.2 & \text { The threshold age for } \bar{H} & 87\end{array}$

$\begin{array}{lll}3 & \text { Related results } & 88\end{array}$

$4 \quad$ Applications $\quad 89$

$4.1 \quad$ Numerical findings $\quad 89$

4.2 The threshold age of the lifetable entropy within the Gompertz mortality model 91

4.3 Decomposition of the relative derivative of $\bar{H} \quad 92$

5 Conclusion $\quad 93$

$\begin{array}{ll}\text { References } & 94\end{array}$

$\begin{array}{ll}\text { Appendix } & 98\end{array}$ 


\title{
The threshold age of the lifetable entropy
}

\author{
José Manuel Aburto ${ }^{1}$ \\ Jesús-Adrián Alvarez ${ }^{2}$ \\ Francisco Villavicencio ${ }^{3}$ \\ James W. Vaupel ${ }^{4}$
}

\begin{abstract}
BACKGROUND

Indicators of relative variation of lifespans are markers of inequality at the population level and of uncertainty at the time of death at the individual level. In particular, the lifetable entropy $\bar{H}$ represents the elasticity of life expectancy to a change in mortality. However, it is unknown how this measure changes over time and whether a threshold age exists, as it does for other lifespan variation indicators.
\end{abstract}

\section{RESULTS}

The time derivative of $\bar{H}$ can be decomposed into changes in life disparity $e^{\dagger}$ and life expectancy at birth $e_{o}$. Likewise, changes over time in $\bar{H}$ are a weighted average of agespecific rates of mortality improvements. These weights reflect the sensitivity of $\bar{H}$ and show how mortality improvements can increase (or decrease) the relative inequality of lifespans. Further, we prove that in the assumption that mortality is reduced at all ages, $\bar{H}$, as well as $e^{\dagger}$, has a threshold age below which saving lives reduces entropy, whereas improvements above that age increase entropy.

\section{CONTRIBUTION}

We give a formal expression for changes of $\bar{H}$ over time and provide a formal proof of the existence of a unique threshold age that separates reductions and increases in lifespan variation as a result age-specific mortality improvements.

\footnotetext{
${ }^{1}$ Interdisciplinary Center on Population Dynamics, University of Southern Denmark, Odense, Denmark and Max Planck Institute for Demographic Research, Rostock, Germany. Email: jmaburto@sdu.dk.

${ }^{2}$ Interdisciplinary Center on Population Dynamics, University of Southern Denmark, Odense, Denmark.

${ }^{3}$ Department of International Health, Bloomberg School of Public Health, Johns Hopkins University, Baltimore, MD, USA.

${ }^{4}$ Interdisciplinary Center on Population Dynamics, University of Southern Denmark, Odense, Denmark, and Max Planck Institute for Demographic Research, Rostock, Germany.
} 


\section{Relationship}

The lifetable entropy is a dimensionless indicator of the relative variation in the length of life compared to life expectancy at birth (Leser 1955; Keyfitz 1968, 1977; Demetrius 1974, 1978). It is usually defined as

$$
\bar{H}(t)=-\frac{\int_{0}^{\infty} \ell(a, t) \ln \ell(a, t) d a}{\int_{0}^{\infty} \ell(a, t) d a}=\int_{0}^{\infty} c(a, t) H(a, t) d a=\frac{e^{\dagger}(t)}{e_{o}(t)},
$$

where $e^{\dagger}(t)=-\int_{0}^{\infty} \ell(a, t) \ln \ell(a, t) d a$ is the life disparity or number of life-years lost as a result of death (Vaupel and Canudas-Romo 2003), $e_{o}(t)=\int_{0}^{\infty} \ell(a, t) d a$ is the life expectancy at birth at time $t, \ell(a, t)$ is the lifetable survival function, $c(a, t)=$ $\ell(a, t) / \int_{0}^{\infty} \ell(x, t) d x$ is the population structure, and $H(a, t)=\int_{0}^{a} \mu(x, t) d x$ is the cumulative hazard to age $a$, where $\mu(x, t)$ is the force of mortality (hazard rate or risk of death) at age $x$ at time $t$. Note that $\bar{H}(t)$ can be interpreted as an average value of $H(a, t)$ in the population at time $t$.

Goldman and Lord (1986) and Vaupel (1986) proved that

$$
e^{\dagger}(t)=\int_{0}^{\infty} d(a, t) e(a, t) d a
$$

where $d(a, t)$ represents the distribution of deaths, and $e(a, t)=\int_{a}^{\infty} \ell(x, t) d x / \ell(a, t)$ is the remaining life expectancy at age $a$ at time $t$. This formulation provides an alternative expression for the lifetable entropy as

$$
\bar{H}(t)=\frac{\int_{0}^{\infty} d(a, t) e(a, t) d a}{\int_{0}^{\infty} \ell(a, t) d a} .
$$

Let $\dot{\bar{H}}$ denote the partial derivative of $\bar{H}$ with respect to time. ${ }^{5}$ We define $\rho(x)=$ $-\dot{\mu}(x) / \mu(x)$ as the age-specific rates of mortality improvements. Then, the relative derivative of $\bar{H}$ can be expressed as a weighted average of $\rho(x)$,

$$
\dot{\bar{H}} / \bar{H}=\int_{0}^{\infty} \rho(x) w(x) W(x) d x
$$

with weights

$$
w(x)=\mu(x) \ell(x) e(x) \quad \text { and } \quad W(x)=\frac{1}{e^{\dagger}}(H(x)+\bar{H}(x)-1)-\frac{1}{e_{o}} .
$$

\footnotetext{
${ }^{5}$ In the following, a dot over a function will denote its partial derivative with respect to time $t$, but variable $t$ will be omitted for simplicity.
} 
Function $\bar{H}(x)$ is the lifetable entropy conditioned on surviving to age $x$, defined as

$$
\bar{H}(x)=\frac{e^{\dagger}(x)}{e(x)}=\frac{\int_{x}^{\infty} d(a) e(a) d a}{\int_{x}^{\infty} \ell(a) d a} .
$$

where $e^{\dagger}(x)=\int_{x}^{\infty} d(a) e(a) d a / \ell(x)$ refers to life disparity above age $x$, and $e(x)$ is the remaining life expectancy at age $x$.

Note that the lifetable entropy $\bar{H}$ is a measure of relative lifespan variation. Thus, higher values represent more variation, whereas lower values denote less variation of lifespans. If mortality improvements over time occur at all ages, there exists a unique threshold age $a^{H}$ that separates positive from negative contributions to the lifetable entropy $\bar{H}$ resulting from those mortality improvements. This threshold age $a^{H}$ is reached when

$$
H\left(a^{H}\right)+\bar{H}\left(a^{H}\right)=1+\bar{H} .
$$

\section{Proof}

Fernández and Beltrán-Sánchez (2015) showed that the relative derivative of $\bar{H}$ can be expressed as

$$
\dot{\bar{H}} / \bar{H}=\frac{\dot{e}^{\dagger}}{e^{\dagger}}-\frac{\dot{e}_{o}}{e_{o}} .
$$

This formula indicates that relative changes in $\bar{H}$ over time are given by the difference between relative changes in $e^{\dagger}$ (dispersion component) and relative changes in $e_{o}$ (translation component). We will first provide expressions for $\dot{e}_{o}$ and $\dot{e}^{\dagger}$ to prove that (1) and (3) are equivalent. Next, we will prove the existence of a threshold age for $\bar{H}$ and its uniqueness.

\subsection{Relative changes over time in $\bar{H}$}

Vaupel and Canudas-Romo (2003) showed that changes over time in life expectancy at birth are a weighted average of the total rates of mortality improvements, given by

$$
\dot{e}_{o}=\int_{0}^{\infty} \rho(x) w(x) d x,
$$

where $\rho(x)=-\dot{\mu}(x) / \mu(x)$ are the age-specific rates of mortality improvements, and $w(x)=\mu(x) \ell(x) e(x)=d(x) e(x)$ is a measure of the importance of death at age $x$. 
Since $d(x)=\mu(x) \ell(x)$ and $\ell(x) e(x)=\int_{x}^{\infty} \ell(a) d a$, the partial derivative with respect to time of $e^{\dagger}=\int_{0}^{\infty} d(x) e(x) d x$ can be expressed as

$$
\begin{aligned}
\dot{e}^{\dagger} & =\int_{0}^{\infty} \dot{\mu}(x) \ell(x) e(x) d x+\int_{0}^{\infty} \mu(x) \int_{x}^{\infty} \dot{\ell}(a) d a d x \\
& =-\int_{0}^{\infty} \rho(x) w(x) d x+\int_{0}^{\infty} \dot{\ell}(a) \int_{0}^{a} \mu(x) d x d a \\
& =-\int_{0}^{\infty} \rho(x) w(x) d x-\int_{0}^{\infty} \int_{0}^{a} \dot{\mu}(x) d x \ell(a) H(a) d a,
\end{aligned}
$$

where $H(a)$ is the cumulative hazard to age $a$. By reversing the order of integration and doing some additional manipulations, we get

$$
\begin{aligned}
\dot{e}^{\dagger} & =-\int_{0}^{\infty} \rho(x) w(x) d x-\int_{0}^{\infty} \dot{\mu}(x) \int_{x}^{\infty} \ell(a) H(a) d a d x \\
& =-\int_{0}^{\infty} \rho(x) w(x) d x+\int_{0}^{\infty} \rho(x) w(x) \frac{\int_{x}^{\infty} \ell(a) H(a) d a}{\ell(x) e(x)} d x \\
& =\int_{0}^{\infty} \rho(x) w(x)\left(\frac{\int_{x}^{\infty} \ell(a)(H(a)-H(x)+H(x)) d a}{\ell(x) e(x)}-1\right) d x \\
& =\int_{0}^{\infty} \rho(x) w(x)\left(H(x) \frac{\int_{x}^{\infty} \ell(a) d a}{\ell(x) e(x)}+\frac{\int_{x}^{\infty} \ell(a)(H(a)-H(x)) d a}{\ell(x) e(x)}-1\right) d x \\
& =\int_{0}^{\infty} \rho(x) w(x)\left(H(x)+\frac{\int_{x}^{\infty} \ell(a)(H(a)-H(x)) d a}{\ell(x) e(x)}-1\right) d x .
\end{aligned}
$$

In Proposition 1 in the Appendix, we prove that

$$
e^{\dagger}(x)=\frac{1}{\ell(x)} \int_{x}^{\infty} d(a) e(a) d a=\frac{1}{\ell(x)} \int_{x}^{\infty} \ell(a)(H(a)-H(x)) d a .
$$

Replacing (6) in (5) yields

$$
\begin{aligned}
\dot{e}^{\dagger} & =\int_{0}^{\infty} \rho(x) w(x)\left(H(x)+\frac{e^{\dagger}(x)}{e(x)}-1\right) d x \\
& =\int_{0}^{\infty} \rho(x) w(x)(H(x)+\bar{H}(x)-1) d x .
\end{aligned}
$$


Finally, replacing the expressions of $\dot{e}_{o}$ and $\dot{e}^{\dagger}$ from (4) and (7) in (3), we get

$$
\begin{aligned}
\dot{\bar{H}} / \bar{H} & =\frac{1}{e^{\dagger}} \int_{0}^{\infty} \rho(x) w(x)(H(x)+\bar{H}(x)-1) d x-\frac{1}{e_{o}} \int_{0}^{\infty} \rho(x) w(x) d x \\
& =\int_{0}^{\infty} \rho(x) w(x)\left(\frac{1}{e^{\dagger}}(H(x)+\bar{H}(x)-1)-\frac{1}{e_{o}}\right) d x \\
& =\int_{0}^{\infty} \rho(x) w(x) W(x) d x,
\end{aligned}
$$

which proves (1) and shows that relative changes over time in the lifetable entropy $\bar{H}$ are the average of the rates of mortality improvements weighted by the product $w(x) W(x)$.

\subsection{The threshold age for $\bar{H}$}

Using (1), changes over time in the lifetable entropy $\bar{H}$ are given by

$$
\dot{\bar{H}}=\bar{H} \int_{0}^{\infty} \rho(x) w(x) W(x) d x .
$$

Whenever $\dot{\bar{H}}>0$, lifespan inequality increases over time, whereas $\dot{\bar{H}}<0$ implies that variation of lifespans decreases over time. Because $\ell(x)$ is a positive function bounded between 0 and 1, we have that $\bar{H}>0$. Moreover, assuming age-specific death rates $\mu(x)$ improve over time at all ages, then $\dot{\mu}(x)<0$ and $\rho(x)>0$ at any age $x$. Therefore, (8) implies that

1. Those ages $x$ in which $w(x) W(x)>0$ will contribute positively to the lifetable entropy $\bar{H}$ and increase lifespan variation;

2. Those ages $x$ in which $w(x) W(x)<0$ will contribute negatively to the lifetable entropy $\bar{H}$ and favor lifespan equality;

3. Those ages $x$ in which $w(x) W(x)=0$ will have no effect on the variation over time of $\bar{H}$.

Our goal is to prove that if mortality improvements occur for all ages and $\rho(x)>0$, there exists a unique threshold age $a^{H}$ such that $w\left(a^{H}\right) W\left(a^{H}\right)=0$. That threshold age will separate positive from negative contributions to $\bar{H}$ resulting from mortality improvements. 
The product $w(x) W(x)$ can be re-expressed as

$$
\begin{aligned}
w(x) W(x) & =\mu(x) \ell(x) e(x)\left(\frac{1}{e^{\dagger}}(H(x)+\bar{H}(x)-1)-\frac{1}{e_{o}}\right) \\
& =\frac{\mu(x) \ell(x) e(x)}{e^{\dagger}}(H(x)+\bar{H}(x)-\bar{H}-1) .
\end{aligned}
$$

Since $\mu(x), \ell(x), e(x)$ and $e^{\dagger}$ are all positive functions, the threshold age of $\bar{H}$ occurs whenever

$$
g(x):=H(x)+\bar{H}(x)-\bar{H}-1=0 .
$$

When $x$ is close to $0, g(x)$ takes negative values since

$$
g(0)=H(0)+\bar{H}(0)-\bar{H}-1=0+\bar{H}-\bar{H}-1=-1<0 .
$$

Likewise, $g(x)$ takes positive values when $x$ becomes arbitrarily large. Note that $\bar{H}$ does not depend on age, and therefore

$$
\lim _{x \rightarrow \infty} g(x)=\lim _{x \rightarrow \infty}(H(x)+\bar{H}(x))-\bar{H}-1=\infty
$$

because $\lim _{x \rightarrow \infty} H(x)=\infty$. By definition, $\bar{H}(x) \geq 0$ for all $x$, so regardless of the behavior of $\bar{H}(x)$, when $x$ is arbitrarily large, the limit of $g(x)$ tends to infinity. Hence, given that $g(0)=-1$ and $\lim _{x \rightarrow \infty} g(x)=\infty$, in a continuous framework the intermediate value theorem guarantees the existence of at least one age $a^{H}$ at which $g\left(a^{H}\right)=0$.

Moreover, as shown in Proposition 2 in the Appendix, $g(x)$ is a strictly increasing function, and therefore a one-to-one function assuming continuity. As a result, there is a unique threshold age $a^{H}$ that separates positive from negative contributions to the lifetable entropy $\bar{H}$, and that threshold age is reached when

$$
w(x) W(x)=0 \Longleftrightarrow g(x)=0 \Longleftrightarrow H(x)+\bar{H}(x)=1+\bar{H},
$$

which proves (2).

\section{Related results}

Demographers have developed a battery of indicators to measure how lifespans vary in populations (van Raalte and Caswell 2013; Colchero et al. 2016). The most common indexes are the variance (Edwards and Tuljapurkar 2005; Tuljapurkar and Edwards 2011), standard deviation (Alvarez, Aburto, and Canudas-Romo 2019; van Raalte, Sasson, and Martikainen 2018) and coefficient of variation (Aburto et al. 2018) of the age at death 
distribution, the Gini coefficient (Shkolnikov, Andreev, and Begun 2003; Gigliarano, Basellini, and Bonetti 2017; Archer et al. 2018), the Theil index (Smits and Monden 2009), and the years of life lost (Vaupel, Zhang, and van Raalte 2011; Aburto and van Raalte 2018), among others. However, only a few studies have analytically derived formulas for the threshold age below and above which mortality improvements respectively decrease and increase lifespan variation. Zhang and Vaupel (2009) showed that the threshold age $\left(a^{\dagger}\right)$ for life disparity $\left(e^{\dagger}\right)$ occurs when $H(x)+\bar{H}(x)=1$. Similarly, Gillespie, Trotter, and Tuljapurkar (2014) determined a threshold age for the variance of the age at death distribution. Van Raalte and Caswell (2013) also showed that it is possible to determine the threshold age by performing an empirical sensitivity analysis of lifespan variation indicators.

In this article, we contribute to the lifespan variation literature by deriving the threshold age $a^{H}$ for the lifetable entropy $\bar{H}$. This age separates negative from positive contributions of age-specific mortality improvements. We analytically proved its existence and - in the assumption that mortality improves over time for all ages - also its uniqueness. In Section 4 we empirically show that it differs from the threshold age of $e^{\dagger}$.

\section{Applications}

The code and data to reproduce the results and graphs presented in this section are publicly available through the repository in the link https://bit.ly/2wqzOFp.

\subsection{Numerical findings}

Figure 1 depicts the threshold ages of the two related measures: life disparity $e^{\dagger}$ and lifetable entropy $\bar{H}$. Calculations were performed using data from the Human Mortality Database (2018) for females in the United States and Italy in 2005. The blue line represents $g(x)$ from Equation (9). The threshold age $a^{H}$ occurs when $g(x)$ crosses zero. The red and grey lines display the same functions that Zhang and Vaupel (2009) used to find the threshold age for $e^{\dagger}$ rescaled to fit in the graph. The intersection of these two lines denotes the threshold age $a^{\dagger}$. Finally, the dashed black line depicts the life expectancy at birth. Vaupel, Zhang, and van Raalte (2011) noted that $a^{\dagger}$ tends to fall just below $e_{o}$. The threshold age for the lifetable entropy $a^{H}$ is greater than $a^{\dagger}$ and is very close above life expectancy for these countries. Note the similarity between the formulas for $a^{\dagger}$, given by $H\left(a^{\dagger}\right)+\bar{H}\left(a^{\dagger}\right)=1$, and $a^{H}$, given by $H\left(a^{H}\right)+\bar{H}\left(a^{H}\right)=1+\bar{H}$. 
Figure 1: $\quad$ Threshold ages for life disparity $\left(a^{\dagger}\right)$ and for the lifetable entropy $\left(a^{H}\right)$, United States and Italy in $\mathbf{2 0 0 5}$

a) United States

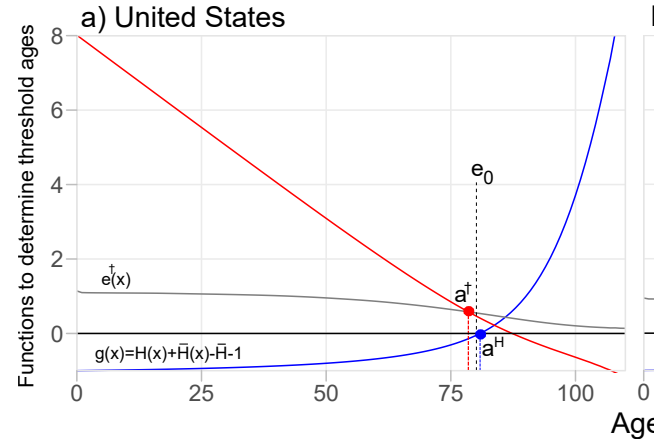

b) Italy

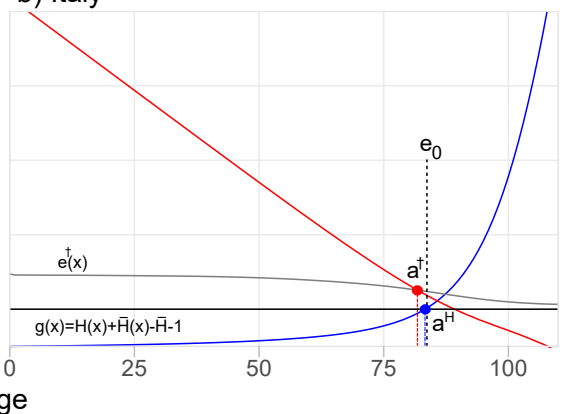

Note: Values in Panel a): $e_{\mathrm{o}}=80.13, a^{\dagger}=78.51$, and $a^{H}=80.86$. Values in Panel b): $e_{o}=83.67, a^{\dagger}=81.76$, and $a^{H}=83.28$. Functions to determine the threshold age for $e^{\dagger}$ were rescaled by a factor of $1 / 10$ for comparability. Source: Human Mortality Database (2018).

Panels a) and b) in Figure 2 illustrate the evolution over time of the threshold ages for $e^{\dagger}$ and $\bar{H}$ in French and Swedish females, respectively. We chose these countries because they portray large series of reliable data available at the Human Mortality Database (2018).

Figure 2: $\quad$ Threshold ages for life disparity $\left(a^{\dagger}\right)$ and for the lifetable entropy $\left(a^{H}\right)$ compared to life expectancy at birth. French and Swedish females, 1800-2016
a) France

b) Sweden

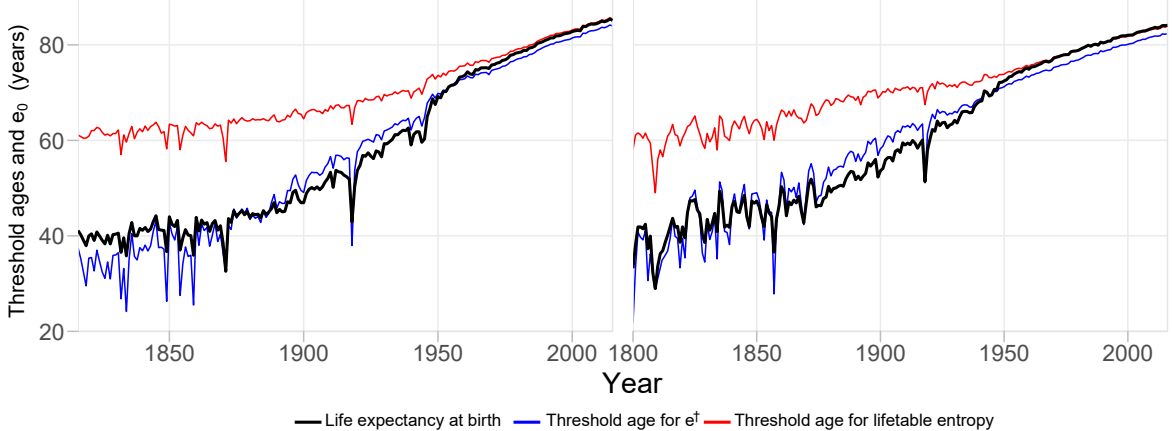

Source: Human Mortality Database (2018).

Values for $a^{\dagger}$ are close to life expectancy throughout the period. However, around 
1950 there is a crossover between $a^{\dagger}$ and $e_{o}$ such that $a^{\dagger}$ remained close to life expectancy, but below it most of the time. This result shows that values of $a^{\dagger}$ below $e_{o}$ is a modern feature of aging populations with high life expectancy. From the beginning of the period of observation to the 1950s, the threshold age for the lifetable entropy was above life expectancy for both countries. During some periods $a^{H}$ was roughly constant whereas $e_{o}$ trended upward. After the 1950s, $a^{H}$ converged toward life expectancy.

\subsection{The threshold age of the lifetable entropy within the Gompertz mortality model}

We further analyze the relationship between $a^{H}$ and $e_{o}$, assuming that the force of mortality follows a Gompertz distribution with hazard $\mu(x)=\alpha \mathrm{e}^{\beta x}$, where $x \geq 0$ denotes the age and $\alpha, \beta>0$ are parameters. In Proposition 3 in the Appendix, we prove that in the Gompertz model, the threshold age $a^{H}$ of the lifetable entropy $\bar{H}$ is approximately proportional to $e_{o}$ by a factor $\delta$, which only depends on parameters $\alpha$ and $\beta$, and the Euler-Mascheroni constant $\gamma \approx 0.57722$. A value of $\delta$ close to 1 indicates that mortality is roughly following a Gompertz model.

Figure 3 shows the evolution over time of factor $\delta$ for French and Swedish females. The observation that this value converges toward 1 could be explanatory for the convergence of the threshold age and life expectancy at birth in modern mortality profiles. It also indicates that modern mortality schedules are roughly Gompertzian. Therefore, it can be speculated that differences between these two measures in earlier years are a consequence of a big proportion of deaths occurring in ages where the force of mortality does not follow a Gompertz, such as in infancy. This pattern is consistent with historical trends which suggest that, from hunter-gathers to modern populations, death rates have decreased at all ages, but mostly at younger ones (Burger, Baudisch, and Vaupel 2012). 
Figure 3:

Factor value $\delta$ for threshold age under Gompertz distribution for French and Swedish women

a) France

b) Sweden

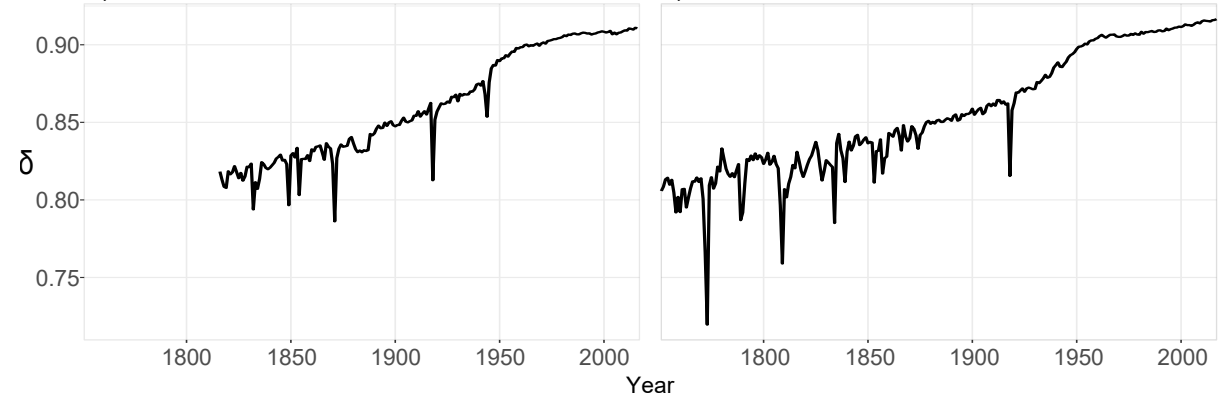

\subsection{Decomposition of the relative derivative of $\bar{H}$}

The relative derivative of $\bar{H}$ defined in Equation (1) can be decomposed between components before and after the threshold age $a^{H}$ as follows:

$$
\begin{aligned}
\dot{\bar{H}} / \bar{H} & =\int_{0}^{\infty} \rho(x) w(x) W(x) d x \\
& =\int_{0}^{a^{H}} \rho(x) w(x) W(x) d x+\int_{a^{H}}^{\infty} \rho(x) w(x) W(x) d x \\
& =\underbrace{\left\{\frac{\dot{e}^{\dagger}\left[x \mid x<a^{H}\right]}{e^{\dagger}}-\frac{\dot{e}_{o}\left[x \mid x<a^{H}\right]}{e_{o}}\right\}}_{\text {Early life component }}+\underbrace{\left\{\frac{\dot{e}^{\dagger}\left[x \mid x>a^{H}\right]}{e^{\dagger}}-\frac{\dot{e}_{o}\left[x \mid x>a^{H}\right]}{e_{o}}\right\}}_{\text {Late life component }}
\end{aligned}
$$

If mortality reductions occur at every age, the early life component in Equation (10) is always negative (contributing to reduce entropy) while the late life component is positive (contributing to increase entropy). Thus, it is clear that a negative relationship between life expectancy and entropy over time occurs if the early life component outpaces the late life component. This decomposition is based on the additive properties of the derivatives of life expectancy and $e^{\dagger}$, as previously shown by Vaupel and Canudas-Romo (2003) and Fernández and Beltrán-Sánchez (2015). 


\section{Conclusion}

Several authors have been interested in decomposing changes over time in life expectancy (Arriaga 1984; Vaupel 1986; Pollard 1988; Vaupel and Canudas-Romo 2003; BeltránSánchez, Preston, and Canudas-Romo 2008; Beltrán-Sánchez and Soneji 2011). Most recently, scholars have also investigated how life disparity fluctuations over time can be decomposed (Zhang and Vaupel 2009; Wagner 2010; Shkolnikov et al. 2011; Aburto and van Raalte 2018; Aburto and Beltrán-Sánchez 2019). Here, we bring both perspectives together and shed light on the dynamics behind changes in the lifetable entropy.

Leser (1955) first derived the lifetable entropy as the elasticity of life expectancy. Keyfitz (1977) proposed $\bar{H}$ as a lifetable function "that measures the change in life expectancy at birth consequent on a proportional change in age-specific rates" (Keyfitz 1977: 413). Since then, several authors have been interested in this measure and its use (Demetrius 1978, 1979; Mitra 1978; Goldman and Lord 1986; Vaupel 1986; Hakkert 1987; Hill 1993; Fernández and Beltrán-Sánchez 2015). Even though the lifetable entropy and $e^{\dagger}$ are both measures of lifespan variation, their demographic interpretation differs. The former is defined as the elasticity of life expectancy due to changes in death rates (Keyfitz 1968) whereas the latter one refers to the average years lost due to death (Vaupel, Zhang, and van Raalte 2011). The life table entropy measures relative variability, while $e^{\dagger}$ measures absolute lifespan variation. Therefore, the lifetable entropy is appropriate to compare different shapes of age-at-death distributions across different species and over time (Baudisch 2011; Wrycza, Missov, and Baudisch 2015), while $e^{\dagger}$ has been used to obtain insights about lifespan variation in different countries and in subpopulation groups, for instance by occupational class or income (van Raalte, Martikainen, and Myrskylä 2014; Brønnum-Hansen 2017). Both measures are meaningful and complementary, but the calculation of their threshold ages should be performed accordingly to correctly interpret changes of age patterns of mortality.

In this article, we uncovered the mathematical regularities behind the changes over time in the lifetable entropy. In particular, this study contributes to the existing literature by showing that (1) the lifetable entropy can be decomposed as a weighted average of rates of mortality improvements, and (2) there exists a unique threshold age that separates positive from negative contributions to lifetable entropy as a result of reductions in mortality over time. 


\section{References}

Aburto, J.M. and Beltrán-Sánchez, H. (2019). Upsurge of homicides and its impact on life expectancy and life span inequality in Mexico, 2005-2015. American Journal of Public Health 109(3): 483-489. doi:10.2105/AJPH.2018.304878.

Aburto, J.M. and van Raalte, A.A. (2018). Lifespan dispersion in times of life expectancy fluctuation: The case of Central and Eastern Europe. Demography 55(6): 2071-2096. doi:10.1007/s13524-018-0729-9.

Aburto, J.M., Wensink, M., van Raalte, A., and Lindahl-Jacobsen, R. (2018). Potential gains in life expectancy by reducing inequality of lifespans in Denmark: An international comparison and cause-of-death analysis. BMC Public Health 18(1): 831. doi:10.1186/s12889-018-5730-0.

Alvarez, J., Aburto, J., and Canudas-Romo, V. (2019). Latin american convergence and divergence towards the mortality profiles of developed countries. Population Studies 1-18. doi:10.1080/00324728.2019.1614651.

Archer, C.R., Basellini, U., Hunt, J., Simpson, S.J., Lee, K.P., and Baudisch, A. (2018). Diet has independent effects on the pace and shape of aging in Drosophila melanogaster. Biogerontology 19(1): 1-12. doi:10.1007/s10522-017-9729-1.

Arriaga, E.E. (1984). Measuring and explaining the change in life expectancies. Demography 21(1): 83-96. doi:10.2307/2061029.

Baudisch, A. (2011). The pace and shape of ageing. Methods in Ecology and Evolution 2(4): 375-382. doi:10.1111/j.2041-210X.2010.00087.x.

Baudisch, A., Salguero-Gómez, R., Jones, O.R., Wrycza, T., Mbeau-Ache, C., Franco, M., and Colchero, F. (2013). The pace and shape of senescence in angiosperms. Journal of Ecology 101(3): 596-606. doi:10.1111/1365-2745.12084.

Beltrán-Sánchez, H., Preston, S.H., and Canudas-Romo, V. (2008). An integrated approach to cause-of-death analysis: Cause-deleted life tables and decompositions of life expectancy. Demographic Research 19(35): 1323-1350. doi:10.4054/DemRes.2008. 19.35 .

Beltrán-Sánchez, H. and Soneji, S. (2011). A unifying framework for assessing changes in life expectancy associated with changes in mortality: The case of violent deaths. Theoretical Population Biology 80(1): 38-48. doi:10.1016/j.tpb.2011.05.002.

Brønnum-Hansen, H. (2017). Socially disparate trends in lifespan variation: A trend study on income and mortality based on nationwide danish register data. BMJ Open 7(5): e014489. doi:10.1136/bmjopen-2016-014489. 
Burger, O., Baudisch, A., and Vaupel, J.W. (2012). Human mortality improvement in evolutionary context. Proceedings of the National Academy of Sciences 109(44): 1821018214. doi:10.1073/pnas.1215627109.

Colchero, F., Rau, R., Jones, O.R., Barthold, J.A., Conde, D.A., Lenart, A., Németh, L., Scheuerlein, A., Schoeley, J., Torres, C., Zarulli, V., Altmann, J., Brockman, D.K., Bronikowski, A.M., Fedigan, L.M., Pusey, A.E., Strier, K.B., Baudisch, A., Alberts, S.C., and Vaupel, J.W. (2016). The emergence of longevous populations. Proceedings of the National Academy of Sciences 113(48): e7681-e7690. doi:10.1073/pnas.1612191113.

Demetrius, L. (1974). Demographic parameters and natural selection. Proceedings of the National Academy of Sciences 71(12): 4645-4647. doi:10.1073/pnas.71.12.4645.

Demetrius, L. (1978). Adaptive value, entropy and survivorship curves. Nature 275: 213-214. doi:10.1038/275213a0.

Demetrius, L. (1979). Relations between demographic parameters. Demography 16(2): 329-338. doi:10.2307/2061146.

Edwards, R.D. and Tuljapurkar, S. (2005). Inequality in life spans and a new perspective on mortality convergence across industrialized countries. Population and Development Review 31(4): 645-674. doi:10.1111/j.1728-4457.2005.00092.x.

Fernández, O.E. and Beltrán-Sánchez, H. (2015). The entropy of the life table: A reappraisal. Theoretical Population Biology 104: 26-45. doi:10.1016/j.tpb.2015.07.001.

Gigliarano, C., Basellini, U., and Bonetti, M. (2017). Longevity and concentration in survival times: The log-scale-location family of failure time models. Lifetime Data Analysis 23(2): 254-274. doi:10.1007/s10985-016-9356-1.

Gillespie, D.O., Trotter, M.V., and Tuljapurkar, S.D. (2014). Divergence in age patterns of mortality change drives international divergence in lifespan inequality. Demography 51(3): 1003-1017. doi:10.1007/s13524-014-0287-8.

Goldman, N. and Lord, G. (1986). A new look at entropy and the life table. Demography 23(2): 275-282. doi:10.2307/2061621.

Hakkert, R. (1987). Life table transformations and inequality measures: Some noteworthy formal relationships. Demography 24(4): 615-622. doi:10.2307/2061396.

Hill, G. (1993). The entropy of the survival curve: An alternative measure. Canadian Studies in Population 20(1): 43-57. doi:10.25336/P6830H.

Human Mortality Database (2018). Human mortality database. [electronic resource]. Berkeley and Rostock: University of California and Max Planck Institute for Demographic Research. http://www.mortality.org. 
Aburto et al.: The threshold age of the lifetable entropy

Keyfitz, N. (1968). Introduction to the mathematics of populations. Reading: AddisonWesley.

Keyfitz, N. (1977). What difference would it make if cancer were eradicated? An examination of the Taeuber paradox. Demography 14(4): 411-418. doi:10.2307/2060587.

Leser, C. (1955). Variations in mortality and life expectation. Population Studies 9(1): 67-71. doi:10.1080/00324728.1955.10405052.

Missov, T.I. and Lenart, A. (2013). Gompertz-Makeham life expectancies: Expressions and applications. Theoretical Population Biology 90: 29-35. doi:10.1016/j.tpb.2013. 09.013.

Mitra, S. (1978). A short note on the Taeuber paradox. Demography 15(4): 621-623. doi:10.2307/2061211.

Pollard, J.H. (1988). On the decomposition of changes in expectation of life and differentials in life expectancy. Demography 25(2): 265-276. doi:10.2307/2061293.

Shkolnikov, V.M., Andreev, E.M., and Begun, A.Z. (2003). Gini coefficient as a life table function: Computation from discrete data, decomposition of differences and empirical examples. Demographic Research 8(11): 305-358. doi:10.4054/DemRes.2003.8.11.

Shkolnikov, V.M., Andreev, E.M., Zhang, Z., Oeppen, J., and Vaupel, J.W. (2011). Losses of expected lifetime in the United States and other developed countries: Methods and empirical analyses. Demography 48(1): 211-239. doi:10.1007/s13524-011-0015-6.

Smits, J. and Monden, C. (2009). Length of life inequality around the globe. Social Science and Medicine 68(6): 1114-1123. doi:10.1016/j.socscimed.2008.12.034.

Tuljapurkar, S. and Edwards, R.D. (2011). Variance in death and its implications for modeling and forecasting mortality. Demographic Research 24(21): 497-526. doi:10.4054/DemRes.2011.24.21.

van Raalte, A.A. and Caswell, H. (2013). Perturbation analysis of indices of lifespan variability. Demography 50(5): 1615-1640. doi:10.1007/s13524-013-0223-3.

van Raalte, A.A., Martikainen, P., and Myrskylä, M. (2014). Lifespan variation by occupational class: compression or stagnation over time? Demography 51(1): 73-95. doi:10.1007/s13524-013-0253-x.

van Raalte, A.A., Sasson, I., and Martikainen, P. (2018). The case for monitoring lifespan inequality. Science 362(6418): 1002-1004. doi:10.1126/science.aau5811.

Vaupel, J.W. (1986). How change in age-specific mortality affects life expectancy. Population Studies 40(1): 147-157. doi:10.1080/0032472031000141896.

Vaupel, J.W. and Canudas-Romo, V. (2003). Decomposing change in life expectancy: A 
bouquet of formulas in honor of Nathan Keyfitz's $90^{\text {th }}$ birthday. Demography 40(2): 201-216. doi:10.1353/dem.2003.0018.

Vaupel, J.W., Zhang, Z., and van Raalte, A.A. (2011). Life expectancy and disparity: An international comparison of life table data. BMJ Open e000128: 1-6. doi:10.1136/bmjopen-2011-000128.

Wagner, P. (2010). Sensitivity of life disparity with respect to changes in mortality rates. Demographic Research 23(3): 63-72. doi:10.4054/DemRes.2010.23.3.

Wrycza, T. (2014). Entropy of the Gompertz-Makeham mortality model. Demographic Research 30(49): 1397-1404. doi:10.4054/DemRes.2014.30.49.

Wrycza, T.F., Missov, T.I., and Baudisch, A. (2015). Quantifying the shape of aging. PLoS ONE 10(3): e0119163. doi:10.1371/journal.pone.0119163.

Zhang, Z. and Vaupel, J.W. (2009). The age separating early deaths from late deaths. Demographic Research 20(29): 721-730. doi:10.4054/DemRes.2009.20.29. 


\section{Appendix}

\section{Proposition 1}

Let $e^{\dagger}(x)=\int_{x}^{\infty} d(a) e(a) d a / \ell(x)$ be a measure of lifespan disparity above age $x$, where $d(a)$ accounts for the distribution of deaths, $e(a)$ the remaining life expectancy at age $a$, and $\ell(x)$ is the probability of surviving from birth to age $x$. Then,

$$
e^{\dagger}(x)=\frac{1}{\ell(x)} \int_{x}^{\infty} \ell(a)(H(a)-H(x)) d a,
$$

where $H(x)$ is the cumulative hazard to age $x$.

Proof. Note that

$$
\frac{1}{\ell(x)} \int_{x}^{\infty} \ell(a)(H(a)-H(x)) d a=\frac{1}{\ell(x)} \int_{x}^{\infty} \ell(a) \int_{x}^{a} \mu(y) d y d a,
$$

where function $\mu(y)$ is the force of mortality or hazard rate. By reversing the order of integration, and using that $e(y)=\int_{y}^{\infty} \ell(a) d a / \ell(y)$ and $d(y)=\mu(y) \ell(y)$, we get

$$
\begin{aligned}
\frac{1}{\ell(x)} \int_{x}^{\infty} \ell(a) \int_{x}^{a} \mu(y) d y d a & =\frac{1}{\ell(x)} \int_{x}^{\infty} \mu(y) \int_{y}^{\infty} \ell(a) d a d y \\
& =\frac{1}{\ell(x)} \int_{x}^{\infty} \mu(y) \ell(y) e(y) d y \\
& =\frac{1}{\ell(x)} \int_{x}^{\infty} d(y) e(y) d y \\
& =e^{\dagger}(x),
\end{aligned}
$$

which proves (A1).

\section{Proposition 2}

Let $\ell(x)$ be the probability of surviving from birth to age $x$. Let $\bar{H}$ be the lifetable entropy and $\bar{H}(x)=e^{\dagger}(x) / e(x)$ the lifetable entropy conditioned on reaching age $x$. Let $H(x)$ be the cumulative hazard to age $x$. Then, $g(x):=H(x)+\bar{H}(x)-1-\bar{H}$ is a strictly increasing function.

Proof. In order to demonstrate that $g(x)$ is a strictly increasing function it is sufficient to show that its first derivative is always positive. We must prove that

$$
\frac{\partial}{\partial x} g(x)=\frac{\partial}{\partial x}(H(x)+\bar{H}(x)-1-\bar{H})=\frac{\partial}{\partial x} H(x)+\frac{\partial}{\partial x} \bar{H}(x)>0
$$


for all ages $x$.

By the fundamental theorem of calculus,

$$
\frac{\partial}{\partial x} H(x)=\frac{\partial}{\partial x} \int_{0}^{x} \mu(a) d a=\mu(x)
$$

whereas

$$
\frac{\partial}{\partial x} \bar{H}(x)=\frac{\partial}{\partial x}\left(\frac{e^{\dagger}(x)}{e(x)}\right)=\frac{1}{e(x)^{2}}\left(e(x) \frac{\partial}{\partial x} e^{\dagger}(x)-e^{\dagger}(x) \frac{\partial}{\partial x} e(x)\right) .
$$

First, note that

(A4)

$$
\begin{aligned}
\frac{\partial}{\partial x} e^{\dagger}(x) & =\frac{\partial}{\partial x}\left(\frac{1}{\ell(x)} \int_{x}^{\infty} d(a) e(a) d a\right) \\
& =\frac{1}{\ell(x)^{2}}\left(\ell(x) \frac{\partial}{\partial x}\left(\int_{x}^{\infty} d(a) e(a) d a\right)-\int_{x}^{\infty} d(a) e(a) d a \frac{\partial}{\partial x} \ell(x)\right) \\
& =\frac{1}{\ell(x)^{2}}\left(\ell(x)(-d(x) e(x))-\int_{x}^{\infty} d(a) e(a) d a(-\mu(x) \ell(x))\right) \\
& =-\frac{\mu(x) \ell(x) e(x)}{\ell(x)}+\mu(x) \frac{\int_{x}^{\infty} d(a) e(a) d a}{\ell(x)} \\
& =\mu(x)\left(e^{\dagger}(x)-e(x)\right) .
\end{aligned}
$$

On the other hand,

$$
\begin{aligned}
\frac{\partial}{\partial x} e(x) & =\frac{\partial}{\partial x}\left(\frac{1}{\ell(x)} \int_{x}^{\infty} \ell(a) d a\right) \\
& =\frac{1}{\ell(x)^{2}}\left(\ell(x) \frac{\partial}{\partial x}\left(\int_{x}^{\infty} \ell(a) d a\right)-\int_{x}^{\infty} \ell(a) d a \frac{\partial}{\partial x} \ell(x)\right) \\
& =\frac{1}{\ell(x)^{2}}\left(\ell(x)(-\ell(x))-\int_{x}^{\infty} \ell(a) d a(-\mu(x) \ell(x))\right) \\
& =e(x) \mu(x)-1 .
\end{aligned}
$$

Therefore, using (A4) and (A5), we get

(A6)

$$
\begin{aligned}
\frac{\partial}{\partial x} \bar{H}(x) & =\frac{1}{e(x)^{2}}\left(e(x) \mu(x)\left(e^{\dagger}(x)-e(x)\right)-e^{\dagger}(x)(e(x) \mu(x)-1)\right) \\
& =\frac{1}{e(x)^{2}}\left(e^{\dagger}(x) e(x) \mu(x)-e(x)^{2} \mu(x)-e^{\dagger}(x) e(x) \mu(x)+e^{\dagger}(x)\right) \\
& =\frac{e^{\dagger}(x)}{e(x)^{2}}-\mu(x) .
\end{aligned}
$$


Finally, replacing (A3) and (A6) in (A2) yields

$$
\frac{\partial}{\partial x} g(x)=\mu(x)+\frac{e^{\dagger}(x)}{e(x)^{2}}-\mu(x)=\frac{e^{\dagger}(x)}{e(x)^{2}}>0,
$$

which holds true for all ages since by definition $e^{\dagger}(x)>0$ for all $x \geq 0$. Hence, $g(x)$ is a strictly increasing function.

\section{Proposition 3}

Assume the force of mortality follows a Gompertz distribution with hazard $\mu(x)=\alpha \mathrm{e}^{\beta x}$, where $x \geq 0$ denotes the age and $\alpha, \beta>0$ are parameters. Suppose mortality improvements over time occur at all ages, therefore there is a unique threshold age $a^{H}$ that separates positive from negative contributions to the lifetable entropy $\bar{H}$. Then, $a^{H}$ is approximately proportional to the life expectancy at birth $e_{o}$.

Proof. The cumulative hazard of the Gompertz model is given by

$$
H(x)=\frac{\alpha}{\beta}\left(\mathrm{e}^{\beta x}-1\right),
$$

where $x \geq 0$ denotes the age and $\alpha, \beta>0$ are parameters. Following Wrycza (2014), the lifetable entropy can be expressed in terms of the Gompertz parameters as

$$
\bar{H}=\frac{1}{\beta}\left(\frac{1}{e_{o}}-\alpha\right),
$$

where $e_{o}$ is the life expectancy at birth. Plugging these two expressions into function $g(x)$ from Equation (9) yields

$$
g(x)=\frac{1}{\beta}\left(\alpha \mathrm{e}^{\beta x}-\frac{1}{e_{o}}\right)+\bar{H}(x)-1 .
$$

From Equation (A1) in Proposition 1, the lifetable entropy conditioned on surviving to age $x$ can be expressed as

$$
\bar{H}(x)=\frac{e^{\dagger}(x)}{e(x)}=\frac{\int_{x}^{\infty} \ell(a)(H(a)-H(x)) d a}{\int_{x}^{\infty} \ell(a) d a} .
$$

Using the above expressions in terms of the Gompertz parameters, it holds that the lifetable entropy conditioned on surviving to age $x$ is

$$
\begin{aligned}
\bar{H}(x) & =\frac{\int_{x}^{\infty} \ell(a) \frac{\alpha}{\beta}\left(\mathrm{e}^{\beta a}-\mathrm{e}^{\beta x}\right) d a}{\int_{x}^{\infty} \ell(a) d a}=\frac{\int_{x}^{\infty} \ell(a) \alpha \mathrm{e}^{\beta a} d a}{\beta \int_{x}^{\infty} \ell(a) d a}-\frac{\alpha}{\beta} \mathrm{e}^{\beta x} \\
& =\frac{\int_{x}^{\infty} \ell(a) \mu(a) d a}{\beta e(x) \ell(x)}-\frac{\alpha}{\beta} \mathrm{e}^{\beta x}=\frac{1}{\beta}\left(\frac{1}{e(x)}-\alpha \mathrm{e}^{\beta x}\right) .
\end{aligned}
$$


The last step in (A8) uses the product $\ell(a) \mu(a)$ as the age-at-death distribution, which then implies that $\int_{x}^{\infty} \ell(a) \mu(a) d a=\ell(x)$. Thus, $g(x)$ in (A7) reduces to

$$
g(x)=\frac{1}{\beta}\left(\frac{1}{e(x)}-\frac{1}{e_{o}}\right)-1,
$$

where $e(x)$ is the remaining life expectancy at age $x$. Equation (A9) implies that the threshold age $a^{H}$ of the lifetable entropy $\bar{H}$ under the Gompertz model occurs whenever

$$
e(x)=\frac{e_{o}}{\beta e_{o}+1} .
$$

Following Missov and Lenart (2013), the remaining life expectancy at age $x$ in the Gompertz case can be approximated by

$$
e(x) \approx \frac{1}{\beta} \mathrm{e}^{\alpha / \beta}(-\gamma-\ln (\alpha / \beta)-\beta x),
$$

where $\gamma \approx 0.57722$ is the Euler-Mascheroni constant. Hence, the threshold age occurs whenever

$$
\begin{aligned}
e(x) & \approx \frac{1}{\beta} \mathrm{e}^{\alpha / \beta}(-\gamma-\ln (\alpha / \beta)-\beta x)=\frac{e_{o}}{\beta e_{o}+1} \\
& \Longleftrightarrow x=-\frac{\mathrm{e}^{-\alpha / \beta} e_{o}}{\beta e_{o}+1}-\frac{1}{\beta}(\gamma+\ln (\alpha / \beta)) .
\end{aligned}
$$
mation,

Note that (A10) implies that $e_{o} \approx \mathrm{e}^{\alpha / \beta}(-\gamma-\ln (\alpha / \beta)) / \beta$. Using this approxi(A11)

$$
\begin{aligned}
a^{H} & \approx-\frac{\mathrm{e}^{-\alpha / \beta} e_{o}}{\mathrm{e}^{\alpha / \beta}(-\gamma-\ln (\alpha / \beta))+1}+\frac{e_{o}}{\mathrm{e}^{\alpha / \beta}} \\
& =\frac{e_{o}}{\mathrm{e}^{\alpha / \beta}}\left(\frac{1}{\mathrm{e}^{\alpha / \beta}(\gamma+\ln (\alpha / \beta))-1}+1\right) \\
& =e_{o}\left(\frac{\gamma+\ln (\alpha / \beta)}{\mathrm{e}^{\alpha / \beta}(\gamma+\ln (\alpha / \beta))-1}\right) \\
& =e_{o} \cdot \delta,
\end{aligned}
$$

which proves that the threshold age $a^{H}$ of the lifetable entropy $\bar{H}$ for the Gompertz model is (approximately) proportional to $e_{o}$ by a factor $\delta$ that only depends on parameters $\alpha, \beta$, and $\gamma$. 
Aburto et al.: The threshold age of the lifetable entropy 